\title{
Submucosal tunneling endoscopic resection of upper gastrointestinal tract tumors arising from muscularis propria
}

\author{
Deepanshu Jain ${ }^{a}$, Aakash Desaib, Ejaz Mahmood ${ }^{a}$, Shashideep Singhal ${ }^{b}$ \\ Albert Einstein Medical Center, Philadelphia; University of Texas Health Science Center at Houston, USA
}

\begin{abstract}
The management of incidentally discovered small upper gastrointestinal (GI) tract submucosal tumors (SMT) remains debatable. In this review, we summarize the evolving experience with submucosal tunneling endoscopic resection (STER) of upper GI SMTs originating from the muscularis propria. From 16 original studies, we reviewed a total of 703 patients with 736 lesions. Of these, 436 were located in the esophagus, 146 in the esophagogastric junction (EGJ) and 154 in the stomach. The composite complete resection rate (CRR) for STER of upper GI tumors arising from the muscularis propria layer was $99.8 \%$ (445/446). The composite CRR for STER of esophageal, EGJ and gastric SMTs arising from the muscularis propria layer was 100\% (208/208),100\% (78/78) and $100 \%$ (115/115), respectively. The composite en bloc resection rate (EBRR) for STER of upper GI tumors arising from the muscularis propria layer was 94.6\% (679/718). The composite EBRR for STER of esophageal, EGJ and gastric SMTs arising from the muscularis propria layer was 98.6\% (205/208), 96.2\% (75/78) and 97.9\% (95/97), respectively. Tumor recurrence rate was 0\%. The reported complication rate for STER was high but the majority responded to conservative management. STER is a minimally invasive and efficacious alternative to surgery, especially for patients with small tumors $(<3 \mathrm{~cm})$. Careful selection of candidates remains crucial for excluding potentially malignant tumors.
\end{abstract}

Keywords Submucosal tunneling endoscopic resection, gastrointestinal tumor, muscularis propria Ann Gastroenterol 2017; 30 (3): 1-11

\section{Introduction}

With the widespread use of endoscopy in routine clinical practice and technological advances in endoscopic procedures and techniques, the incidence of gastric submucosal tumors has increased [1]. Upper gastrointestinal (GI) submucosal tumors, especially those $<3 \mathrm{~cm}$, are mostly benign in nature [2]. However, some tumors, such as mesenchymal neoplasms (including GI stromal tumors originating from the muscularis propria layer), can turn malignant [3]. In order to obtain an accurate diagnosis, needle biopsy is the first step.

${ }^{a}$ Department of Internal Medicine, Albert Einstein Medical Center, Philadelphia, PA (Deepanshu Jain, Ejaz Mahmood); ${ }^{\mathrm{b}}$ Division of Gastroenterology, Hepatology and Nutrition, University of Texas Health Science Center at Houston, Houston, Texas (Aakash Desai, Shashideep Singhal), USA

\section{Conflict of Interest: None}

Correspondence to: Shashideep Singhal, MD, Division of Gastroenterology, Hepatology and Nutrition, University of Texas Health Science Center at Houston, 6431 Fannin, MSB 4.234, Houston, Texas, 77030, USA, e-mail: sdsinghal@gmail.com

Received 06 September 2016; accepted 18 December 2016; published online February 10, 2017

DOI: https://doi.org/10.20524/aog.2017.0128
However, given the possibility of sampling errors when biopsy specimens are obtained, the malignant potential of the tumor cannot be ruled out completely [3]. Thus, in this case periodic endoscopic observations and/or endoscopic ultrasound (EUS) or resection are the only treatment options available for an asymptomatic GI submucosal tumor [4]. Patients generally opt for tumor resection because of issues with the cost, compliance, stress and the risk associated with repeated endoscopic procedures [5].

Various modalities exist for tumor resection, including surgical procedures (open, laparoscopic or thoracoscopic surgery) [6] and newer endoscopic techniques. Endoscopic submucosal dissection (ESD) has emerged as a potential endoscopic technique in patients with small GI submucosal tumors. However, this technique still has some risk of complications, such as perforation, massive bleeding and incomplete resection, especially for tumors that arise from the muscularis propria layer [5,7].

Submucosal tunneling endoscopic resection (STER) was inspired by ESD as a new technique for resection of upper GI submucosal tumors. In this technique, a submucosal tunnel is created to serve as a working space for endoscope insertion and resection of the tumor. This technique has a lower risk of perforation, since the integrity of GI mucosa is maintained; it offers better wound healing and a lower risk of infection when compared to ESD. Furthermore, this method is better suited 
for tumors arising from the muscularis propria layer, for which ESD resection is difficult because of the deeper tumor origin.

In this review, we summarize the safety and efficacy data on the usage of the STER technique for the resection of upper GI tumors arising from the muscularis propria layer. The indications, techniques, procedure time, length of hospital stay and complications are also discussed.

\section{Materials and methods}

An extensive search of the English-language literature up to February 2016 was performed using PubMed and Google Scholar to identify peer-reviewed original and review articles. The keywords used were "submucosal tunneling endoscopic resection", "gastrointestinal tumor", and "muscularis propria". Only human studies were included. The references of pertinent studies were manually searched to identify additional relevant studies. The indications, procedural details, success rates, clinical outcomes, complications and limitations were considered. The methodology for the selection of studies for our review is shown in Fig. 1.

\section{Results}

Seventeen original articles were considered appropriate for inclusion in this review [8-24]. Two articles [14,24] were from same institute and the time frames of these studies overlapped. Hence, only the study [14] with the longer time interval was included in our review and the other study [24] was excluded to avoid duplication of data. Of the remaining 16 studies,

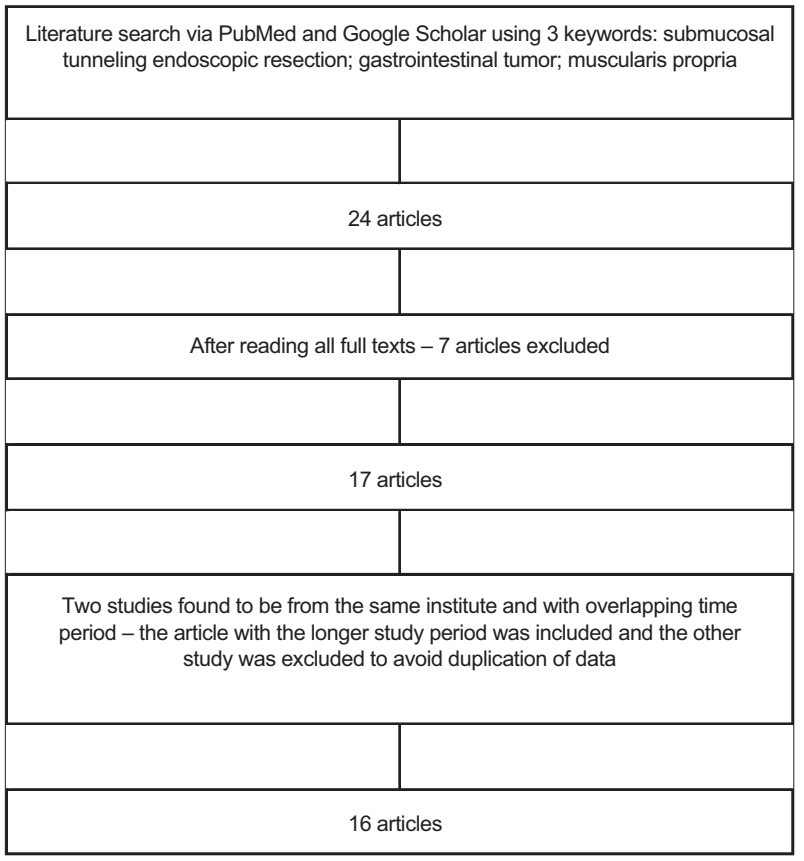

Figure 1 Methodology for selection of articles for review three were case reports [13,16,22], nine were retrospective studies $[8,11,12,14,15,17-19,21]$ and four were prospective studies $[9,10,20,23]$. All studies except two $[16,23]$ were conducted in China [8-15,17-22], which could have led to bias in our results. The studies included only patients with tumors arising from the esophagus [21,22], EGJ [19,20], stomach [16-18], or a mix of those locations $[8-15,23]$. The studies are summarized in Table 1.

\section{Inclusion criteria}

\section{Esophageal/EGJ origin}

Of the four studies, only two clearly described the inclusion criteria for the procedure [19-22]. All subjects had a tumor originating from the muscularis propria layer with a size cutoff ranging from $3.5 \mathrm{~cm} \mathrm{[20]} \mathrm{to} 5.5 \mathrm{~cm} \mathrm{[21],} \mathrm{as} \mathrm{confirmed}$ by EUS and CT scan. In the study by Tan et al, all the patients had Zubrod-Eastern Cooperative Oncology Group (ECOG) performance status (this scale runs from 0 to 4 , with 0 being fully functional and asymptomatic, and 4 being bedridden) of 0 or 1 and a histologically confirmed diagnosis of leiomyoma [21].

\section{Gastric origin}

Most of the studies included patients with a tumor originating from the muscularis propria layer with a size of $<3 \mathrm{~cm}$ and absence of any high-risk features on EUS [16-18].

\section{Mixed}

Tumors originated from the muscularis propria layer, with a size ranging from $1-7.5 \mathrm{~cm}[9,23]$; there was no evidence of any extra luminal growth $[8,14,20]$, tumor or high risk features on EUS $[9,12]$. Innou et al had to offer surgical resection to 2 subjects (out of the initial 9) as there was limited endoscopic visualization due to a large tumor size $(6.0 \mathrm{~cm}$ and $7.5 \mathrm{~cm})$ [23].

\section{Exclusion criteria}

In only 6 of the 15 reviewed studies were the exclusion criteria clearly defined. Common exclusion criteria included intolerance of anesthesia, refusal of consent, or blood coagulation disorders $[9,12,14,20]$. One study excluded patients with cardiovascular disease and those who had tumors in the gastric fundus or lesser curvature of the gastric antrum [12]. A few studies also included a tumor size greater than $3 \mathrm{~cm}$ among their exclusion criteria $[14,15,20]$.

\section{Tumor characteristics}

Among 703 patients with 736 lesions, there were 436 esophageal, 146 EGJ and 154 gastric SMTs arising from the muscularis propria layer of the GI tract. The size of 
tumors originating in the esophagus or EGJ ranged from $0.6 \mathrm{~cm}$ to $5.5 \mathrm{~cm}[13,19-23]$, while for tumors originating in the gastric region the size ranged between $0.8 \mathrm{~cm}$ and $5.0 \mathrm{~cm}[13,16-18,23]$. The other seven studies reported a single mean tumor size irrespective of the location, i.e. esophageal or gastric; hence, the results are not reported together with the above findings $[8-12,14,15]$. Overall [8-23], the authors reported STER for upper GI tract SMTs arising from the muscularis propria layer with sizes varying from as small as $0.6 \mathrm{~cm} \mathrm{[20]} \mathrm{to} \mathrm{as} \mathrm{large} \mathrm{as} 7.0 \mathrm{~cm}$ [14].

The majority of resected tumors were leiomyomas [8-23] or GI stromal tumors (GIST) [8-10,12,14,15,17-20,23], with the minority including calcifying fibrous tumors $[9,14,18]$, schwannomas $[14,15,20]$, nerve sheath tumors [18], glomus tumors [10,14,18], intramuscular lipomas [20], aberrant pancreas [23], or granular cell tumors [20]. The heterogeneity among the tumor characteristics of patients across the individual studies is summarized in Table 1.

\section{Technique}

A standard single accessory channel gastroscope (GIFQ260J), a dual-channel gastroscope (GIF-2T240, Olympus), or a transparent cap (D-201-11802, Olympus) were the devices used by most physicians to perform the procedure [13-23]. One study described the use of a dual-channel endoscope (GIF-Q260J; Olympus) with a plastic fitted cap (MH-583; Olympus) [16].

All procedures were performed under general anesthesia and required a skilled and experienced endoscopic surgeon. Only one study described the experience of the performing endoscopic surgeon in terms of the number of ESD procedures conducted in the past [8].

In a porcine model, the ESD technique was used to create a submucosal tunnel and was shown to be a technically feasible and an effective access method for natural orifice transluminal endoscopic surgery (NOTES) [25]. This technique has been the basis for peroral endoscopic myotomy (POEM) for esophageal achalasia [26]. STER is a relatively new technique inspired from ESD and POEM for the treatment of upper GI tumors. A mucosal incision is made approximately $5 \mathrm{~cm}$ proximal to the SMT, followed by injection of dilute indigo carmine or methylene blue dye to create a mucosal bleb. A 2-cm incision is made at the top of the mucosal bleb [10]. This is followed by a submucosal tunnel, which is created by dissecting the muscle fibers [10]. Once the tumor is located, tumor enucleation is carried out under direct endoscopic visualization using an insulated tip knife, hook knife or hybrid knife depending on the surgeon's preference [10]. After tumor resection, the sub-mucosal tunnel is lavaged with normal saline and hemostasis is obtained [10]. Finally, the mucosal incision site is closed with 4-6 hemostatic clips.

\section{Procedure time}

For tumors of esophageal or EGJ origin, the mean procedure time was $120.1 \mathrm{~min}$ (range: 15-365 min) [19-23]. For gastric tumors, the mean procedure time was shorter at $86.7 \mathrm{~min}$ (range: $25-320 \mathrm{~min}$ ) [16-18,23]. Overall, the procedure time for STER of SMTs arising from the muscularis propria layer of the upper GI tract ranged from as short as $15 \mathrm{~min}[14,20]$ to as long as $365 \mathrm{~min}$ [23].

A few subjects had more than one lesion, which would result in a longer total procedure time and could thus potentially create bias in the interpretation of above results. Details of the procedure times for each individual study are summarized in Table 1.

\section{Complication rate}

There was a wide variation in the rate of reported complications across the studies, varying from as low as $0 \%[13,16,22,23]$ to as high as $42.9 \%$ [19]. The most common complications found in the studies were pneumothorax, subcutaneous emphysema, pneumomediastinum, pneumoperitoneum and pleural effusion [8-12,14,15,17-21]. Mucosal tunnel perforation and chest pain were reported in a few cases [12]. Esophageal fistula and diverticulum were rare [14]. The majority of the complications were managed conservatively with good outcomes [8-12,14,15,17-21]. In the study by Chen et al, the composite complication rate was $23.4 \%$ but only $10 \%$ of the procedures required an intervention for the management of complications [14]. Likewise, Wang et al reported a complication rate of $8.8 \%$, but none of the patients required a repeat surgical intervention [12].

A few common adverse events, such as pneumothorax, pneumomediastinum, pneumoperitoneum and subcutaneous emphysema, are really consequences of the technique and not complications, as reported by authors from different studies. To give a better understanding of the true complication rate, we have compiled the individual incidence rate for adverse events across each study in Table 1 .

\section{Post-procedure discharge instructions}

Postoperative discharge instructions were provided for the patients in order to promote faster healing and reduce complications. In general, patients were advised to remain on nil per os for at least $24 \mathrm{~h}$ before resuming their normal diet $[9,12-14,16,18-21]$. Some studies instructed the patients to remain on only a liquid diet for 3 days before gradually returning to their normal diet over 2 weeks $[12,13,21]$. In one study, subjects underwent endoscopy the day after the procedure and a contrast study to rule out leakage before oral nutrition was permitted [23]. Apart from dietary instructions, patients were also prescribed intravenous/ oral proton pump Inhibitors during and after the operation for a period of 3 days to around 4 weeks, depending on the surgeon's preference [8,9,12-21]. Antibiotics were also prescribed, usually for 3 days, to prevent any postoperative infections [12-15,18,20,21]. No specific choice of antibiotic therapy was mentioned in any of the studies. A few studies also prescribed homeostatic agents to prevent postoperative bleeding and ensure early recovery $[8,17,18,20]$. 
Table 1 Summary of individual studies

\begin{tabular}{|c|c|c|c|c|c|c|c|}
\hline $\begin{array}{l}\text { Author/Year/ } \\
\text { Location }\end{array}$ & Study type & $\begin{array}{l}\text { Inclusion } \\
\text { criteria }\end{array}$ & $\begin{array}{c}\text { Number } \\
\text { of } \\
\text { subjects }\end{array}$ & $\begin{array}{c}\text { Number } \\
\text { of } \\
\text { lesions }\end{array}$ & $\begin{array}{l}\text { Distribution } \\
\text { of lesions }\end{array}$ & $\begin{array}{c}\text { Mean size of } \\
\text { lesion } \\
\text { (range) }\end{array}$ & $\begin{array}{l}\text { Pathologic } \\
\text { diagnosis }\end{array}$ \\
\hline $\begin{array}{l}\text { Lu et al 2014, } \\
\text { China [8] }\end{array}$ & $\begin{array}{l}\text { Single-center, } \\
\text { retrospective } \\
\text { study }\end{array}$ & $\begin{array}{l}\text { 1. Upper GI } \\
\text { submucosal } \\
\text { tumor (SMT) } \\
\text { originating } \\
\text { from muscularis } \\
\text { propria (MP) } \\
\text { layer between } \\
2010-2014 \text {, } \\
\text { confirmed by } \\
\text { endoscopic } \\
\text { ultrasound } \\
\text { (EUS) } \\
2 \text {. Size: } \\
<3 \text { cm without } \\
\text { extraluminal } \\
\text { involvement } \\
\text { confirmed by } \\
\text { CT or S? }\end{array}$ & 42 & 45 & $\begin{array}{l}\text { 1. Esophageal: } \\
29 \\
\text { 2. Gastric: } 16\end{array}$ & $1.2(0.8-1.6)$ & $\begin{array}{l}\text { 1. Leiomyoma } \\
\text { 2. Gastrointestina } \\
\text { stromal tumors } \\
\text { (GIST) }\end{array}$ \\
\hline $\begin{array}{l}\text { Ye et al 2013, } \\
\text { China [9] }\end{array}$ & $\begin{array}{l}\text { Single-center, } \\
\text { prospective } \\
\text { study }\end{array}$ & $\begin{array}{l}\text { 1. SMT } \\
\text { originating } \\
\text { from } \\
\text { MP layer } \\
\text { confirmed by } \\
\text { EUS and CT } \\
\text { 2. Size: } \\
<1-3 \mathrm{~cm} \text { with } \\
\text { no high risk } \\
\text { features on } \\
\text { EUS }\end{array}$ & 85 & 85 & $\begin{array}{l}\text { 1. Esophageal: } \\
60 \\
\text { 2. Gastric: } 25\end{array}$ & $1.9(1.0-3.0)$ & $\begin{array}{l}\text { 1. Leiomyoma } \\
\text { 2. GIST } \\
\text { 3. Calcifying } \\
\text { fibrous tumor }\end{array}$ \\
\hline
\end{tabular}

\begin{tabular}{|c|c|c|c|c|c|c|c|}
\hline $\begin{array}{l}\text { Xu et al 2011, } \\
\text { China [10] }\end{array}$ & $\begin{array}{l}\text { Single-center, } \\
\text { prospective } \\
\text { study }\end{array}$ & $\begin{array}{l}\text { Upper GI SMTs } \\
\text { originating } \\
\text { from MP layer } \\
\text { between June } \\
2010 \text { and March } \\
2011\end{array}$ & 15 & 15 & $\begin{array}{l}\text { 1. Esophageal: } \\
9 \\
\text { 2. Gastric: } 6\end{array}$ & $1.9(1.2-3.0)$ & $\begin{array}{l}\text { 1. Leiomyoma } \\
\text { 2. GIST } \\
\text { 3. Glomus tumor }\end{array}$ \\
\hline
\end{tabular}

\begin{tabular}{|c|c|c|c|c|c|c|c|}
\hline $\begin{array}{l}\text { Zhang et al 2014, } \\
\text { China [11] }\end{array}$ & $\begin{array}{l}\text { Single-center, } \\
\text { retrospective } \\
\text { study }\end{array}$ & $\begin{array}{l}\text { Upper GI SMT } \\
\text { arising from MP } \\
\text { layer confirmed } \\
\text { on EUS }\end{array}$ & 23 & 49 & $\begin{array}{l}\text { 1. Esophageal: } \\
42 \\
\text { 2. Gastric: } 7\end{array}$ & $1.5(0.8-3.5)$ & Leiomyoma \\
\hline
\end{tabular}




\begin{tabular}{|c|c|c|c|c|c|}
\hline $\begin{array}{l}\text { Mean procedure } \\
\text { time (min) } \\
\text { (range) }\end{array}$ & Complications & $\begin{array}{l}\text { Follow-up } \\
\text { interval and } \\
\text { modality }\end{array}$ & $\begin{array}{l}\text { Complete } \\
\text { resection rate } \\
\text { (absolute } \\
\text { number) }\end{array}$ & $\begin{array}{l}\text { Tumor } \\
\text { recurrence } \\
\text { rate }\end{array}$ & $\begin{array}{l}\text { En bloc } \\
\text { resection rate } \\
\text { (absolute } \\
\text { number) }\end{array}$ \\
\hline $\begin{array}{c}84.4 \\
(55.3-113.5)\end{array}$ & $\begin{array}{l}\text { Composite complication rate: data } \\
\text { not available (DNA) } \\
\text { a. Perforation: } 6 / 45(13.3 \%) \\
\text { b. Air leakage: } 1 / 45(2.2 \%)\end{array}$ & $\begin{array}{l}\text { 1. Surveillance } \\
\text { endoscopy at } \\
2 \text { month }(\mathrm{m}) \\
\text { and } 6 \mathrm{~m} \text { post } \\
\text { procedure } \\
\text { and annually } \\
\text { thereafter } \\
\text { 2. Mean follow } \\
\text { up: } 8.7 \mathrm{~m}\end{array}$ & $\begin{array}{l}\text { Composite: } \\
97.7 \%(44 / 45)\end{array}$ & $0 \%$ & $\begin{array}{l}\text { Composite: } \\
97.7 \% \\
(44 / 45)\end{array}$ \\
\hline $\begin{array}{c}57.2 \\
(30-115)\end{array}$ & $\begin{array}{l}\text { Composite complication rate: } 9.4 \% \\
\text { a. Pneumothorax: } 6 / 85(7.1 \%) \\
\text { b. Subcutaneous emphysema: } \\
8 / 84(9.5 \%) \\
\text { c. Pneumoperitoneum: } 4 / 85(4.7 \%)\end{array}$ & $\begin{array}{l}\text { 1. Surveillance } \\
\text { endoscopy at } \\
1,3,6 \mathrm{~m} \text { and } \\
\text { EUS for residual } \\
\text { tumor at } 3 \mathrm{~m} \\
\text { 2. Follow-up } \\
\text { range: } 2-19 \mathrm{~m} \\
3 . \text { For GIST: In } \\
\text { addition to EUS } \\
\text { and endoscopy } \\
\text { for local } \\
\text { recurrence, } \\
\text { annual US } \\
\text { abdomen, } \\
\text { CT scan and } \\
\text { CXR annually } \\
\text { for distant } \\
\text { metastasis } \\
\text { (indefinitely) }\end{array}$ & $\begin{array}{l}\text { 1.Esophageal: } \\
100 \%(60 / 60) \\
\text { 2. Gastric: } \\
100 \%(25 / 25)\end{array}$ & $0 \%$ & $\begin{array}{l}\text { 1. Esophageal: } \\
100 \% \\
(60 / 60) \\
\text { 2. Gastric: } \\
100 \% \\
(25 / 25)\end{array}$ \\
\hline $\begin{array}{c}78.7 \\
(25-130)\end{array}$ & $\begin{array}{l}\text { Composite complication rate: } 13.3 \% \\
\text { a. Pneumothorax and subcutaneous } \\
\text { emphysema: } 1 / 15(6.7 \%) \\
\text { c. Pneumoperitoneum: } 1 / 15(6.7 \%)\end{array}$ & $\begin{array}{l}\text { 1. Surveillance } \\
\text { endoscopy and } \\
\text { EUS at } 1,2,4 \\
\text { and } 6 \mathrm{~m} \text { to } \\
\text { assess healing } \\
\text { and check for } \\
\text { residual tumors } \\
2 \text {. Follow-up } \\
\text { range: } 1-6 \mathrm{~m}\end{array}$ & $\begin{array}{l}\text { 1.Esophageal: } \\
100 \%(9 / 9) \\
\text { 2. Gastric: } \\
100 \%(6 / 6)\end{array}$ & $0 \%$ & $\begin{array}{l}\text { 1. Esophageal: } \\
\text { 100\% (9/9) } \\
\text { 2. Gastric: } \\
100 \%(6 / 6)\end{array}$ \\
\hline $\begin{array}{c}40 \\
(20-75)\end{array}$ & $\begin{array}{l}\text { Composite complication rate: DNA } \\
\text { a. Pneumothorax: } 2 / 23(8.7 \%) \\
\text { b. Subcutaneous emphysema: } \\
3 / 23(13.0 \%) \\
\text { c. Pneumomediastinum and } \\
\text { pneumoperitoneum: } 1 / 23(4.3 \%) \\
\text { d. Thoracic effusion: } 2 / 23(8.7 \%) \\
\text { All complications were managed } \\
\text { conservatively. }\end{array}$ & $\begin{array}{l}\text { Follow-up } \\
\text { range: } \\
3-36 \mathrm{~m} \\
\text { (Median: } 18 \mathrm{~m} \text { ) }\end{array}$ & $\begin{array}{l}\text { 1.Esophageal: } \\
\text { 100\% }(42 / 42) \\
\text { 2. Gastric: } \\
100 \%(7 / 7)\end{array}$ & $0 \%$ & $\begin{array}{l}\text { 1. Esophageal: } \\
100 \% \\
(42 / 42) \\
\text { 2. Gastric: } \\
100 \%(7 / 7)\end{array}$ \\
\hline
\end{tabular}


Table 1 Continued...

\begin{tabular}{|c|c|c|c|c|c|c|c|}
\hline $\begin{array}{l}\text { Author/Year/ } \\
\text { Location }\end{array}$ & Study type & $\begin{array}{l}\text { Inclusion } \\
\text { criteria }\end{array}$ & $\begin{array}{l}\text { Number } \\
\text { of } \\
\text { subjects }\end{array}$ & $\begin{array}{c}\text { Number } \\
\text { of } \\
\text { lesions }\end{array}$ & $\begin{array}{l}\text { Distribution } \\
\text { of lesions }\end{array}$ & $\begin{array}{c}\text { Mean size of } \\
\text { lesion } \\
\text { (range) }\end{array}$ & $\begin{array}{l}\text { Pathologic } \\
\text { diagnosis }\end{array}$ \\
\hline $\begin{array}{l}\text { Wang et al } \\
\text { 2015, China [12] }\end{array}$ & $\begin{array}{l}\text { Single-center, } \\
\text { retrospective } \\
\text { study }\end{array}$ & $\begin{array}{l}\text { 1. SMT } \\
\text { originating from } \\
\text { the MP layer } \\
\text { confirmed by CT } \\
\text { and EUS } \\
\text { 2. EUS shows no } \\
\text { high risk features } \\
\text { of malignancy } \\
\text { 3. No signs of } \\
\text { metastasis or } \\
\text { invasion outside } \\
\text { digestive tract } \\
\text { during CT } \\
\text { 4. Age between } \\
\text { 18-70 years and } \\
\text { Zubrod-ECOG } \\
\text { Performance } \\
\text { status } 0 \text { or } 1\end{array}$ & 80 & 83 & $\begin{array}{l}\text { 1. Esophageal: } \\
67 \\
\text { 2. Gastric: } 16\end{array}$ & $2.3(1.0-5.5)$ & $\begin{array}{l}\text { 1. Leiomyoma } \\
\text { 2. GIST }\end{array}$ \\
\hline $\begin{array}{l}\text { Chen et al 2015, } \\
\text { China [13] }\end{array}$ & Case report & $\begin{array}{l}\text { Upper GI SMT } \\
\text { arising from MP } \\
\text { layer confirmed } \\
\text { on EUS }\end{array}$ & 1 & 2 & $\begin{array}{l}\text { 1. Esophageal: } \\
1 \\
\text { 2. Gastric: } 1\end{array}$ & $\begin{array}{l}\text { 1.Esophagus: } \\
2.5 \times 1.2 \mathrm{~cm} \\
2 . \text { Gastric: } \\
3 \times 1.5 \mathrm{~cm}\end{array}$ & Leiomyoma \\
\hline $\begin{array}{l}\text { Chen et al 2016, } \\
\text { China [14] }\end{array}$ & $\begin{array}{l}\text { Retrospective } \\
\text { study }\end{array}$ & $\begin{array}{l}\text { SMT originated } \\
\text { from the MP } \\
\text { layer without } \\
\text { restriction of } \\
\text { extraluminal } \\
\text { growth }\end{array}$ & 290 & 290 & $\begin{array}{l}\text { 1. Esophagus: } \\
\text { 199 } \\
\text { 2. EGJ: } 68 \\
\text { 3. Gastric: } 23\end{array}$ & $2.1(1.0-7.0)$ & $\begin{array}{l}\text { 1. Leiomyoma } \\
\text { 2. GIST } \\
\text { 3. Calcifying } \\
\text { fibrous tumor } \\
\text { 4. Schwannomas } \\
\text { 5. Glomus tumor }\end{array}$ \\
\hline $\begin{array}{l}\text { Liu et al 2013, } \\
\text { China [15] }\end{array}$ & $\begin{array}{l}\text { Retrospective } \\
\text { study }\end{array}$ & $\begin{array}{l}\text { Upper GI SMT } \\
\text { arising from MP } \\
\text { layer confirmed } \\
\text { on EUS }\end{array}$ & 12 & 12 & $\begin{array}{l}\text { 1. Esophageal: } \\
7 \\
\text { 2. Gastric: } 5\end{array}$ & $1.9(1.0-3.0)$ & $\begin{array}{l}\text { 1. Leiomyoma } \\
\text { 2. GIST } \\
\text { 3. Schwannoma }\end{array}$ \\
\hline $\begin{array}{l}\text { Jeong et al 2015, } \\
\text { Korea [16] }\end{array}$ & Case report & $\begin{array}{l}\text { 1. SMT } \\
\text { originating } \\
\text { predominantly } \\
\text { from the MP } \\
\text { layer, confirmed } \\
\text { on EUS } \\
\text { 2. Size: } 2 \mathrm{~cm}\end{array}$ & 1 & 1 & Gastric: 1 & 2.5 & Leiomyoma \\
\hline $\begin{array}{l}\text { Lu et al 2014, } \\
\text { China [17] }\end{array}$ & $\begin{array}{l}\text { Retrospective } \\
\text { study }\end{array}$ & $\begin{array}{l}\text { 1. SMT } \\
\text { originating } \\
\text { from MP layer, } \\
\text { confirmed on } \\
\text { EUS } \\
2 . \text { Size: } 0.8-5.0 \mathrm{~cm}\end{array}$ & 18 & 18 & Gastric: 18 & $2.1(0.8-5.0)$ & $\begin{array}{l}\text { 1. Leiomyoma } \\
\text { 2. GIST }\end{array}$ \\
\hline
\end{tabular}




\begin{tabular}{|c|c|c|c|c|c|}
\hline $\begin{array}{l}\text { Mean procedure } \\
\text { time (min) } \\
\text { (range) }\end{array}$ & Complications & $\begin{array}{l}\text { Follow-up } \\
\text { interval and } \\
\text { modality }\end{array}$ & $\begin{array}{l}\text { Complete } \\
\text { resection rate } \\
\text { (absolute } \\
\text { number) }\end{array}$ & $\begin{array}{l}\text { Tumor } \\
\text { recurrence } \\
\text { rate }\end{array}$ & $\begin{array}{l}\text { En bloc } \\
\text { resection rate } \\
\text { (absolute } \\
\text { number) }\end{array}$ \\
\hline $\begin{array}{c}61.2 \\
(25-160)\end{array}$ & $\begin{array}{l}\text { Composite complication rate: } \\
8.8 \% \text { (none required surgical } \\
\text { intervention) } \\
\text { a. Pneumothorax: } 1 / 80(1.3 \%) \\
\text { b. Subcutaneous emphysema: } \\
2 / 80(2.5 \%) \\
\text { b. Mucosal perforation of tunnel: } \\
1 / 80(1.3 \%) \\
\text { c. Chest pain: } 3 / 80(3.8 \%)\end{array}$ & $\begin{array}{l}\text { 1. Surveillance } \\
\text { endoscopy at } \\
1,3,6 \text { and } 12 \mathrm{~m} \\
\text { and annually } \\
\text { thereafter. EUS } \\
\text { performed at } 1 \\
\text { and } 12 \mathrm{~m} \\
2 . \text { Follow-up } \\
\text { range: } \\
1-33 \mathrm{~m} \text { (Mean: } \\
10.2 \mathrm{~m} \text { ) }\end{array}$ & $\begin{array}{l}\text { 1. Esophageal: } \\
100 \%(67 / 67) \\
\text { 2. Gastric: } \\
100 \%(16 / 16)\end{array}$ & $0 \%$ & $\begin{array}{l}\text { 1. Esophageal: } \\
98.5 \%(66 / 67) \\
\text { 2. Gastric: } \\
93.8 \%(15 / 16)\end{array}$ \\
\hline DNA & Composite complication rate: $0 \%$ & $\begin{array}{l}\text { Endoscopy at } \\
\text { day } 6 \text { and } 1 \mathrm{~m}\end{array}$ & $\begin{array}{l}\text { 1. Esophageal: } \\
100 \%(1 / 1) \\
\text { 2. Gastric: } \\
100 \%(1 / 1)\end{array}$ & $0 \%$ & $\begin{array}{l}\text { 1. Esophageal } \\
100 \%(1 / 1) \\
\text { 2. Gastric: } \\
100 \%(1 / 1)\end{array}$ \\
\hline $\begin{array}{c}43 \\
(15-200)\end{array}$ & $\begin{array}{l}\text { Composite complication rate: } \\
23.4 \% \text { ( } 10 \% \text { of procedures required } \\
\text { intervention for the complication) } \\
\text { a. Subcutaneous emphysema: } \\
61 / 290(21.0 \%) \\
\text { b. Pneumothorax: } 22 / 290(7.6 \%) \\
\text { c. Pneumoperitoneum: } 15 / 290(5.2 \%) \\
\text { d. Mucosal injury: } 3 / 290(1.0 \%) \\
\text { e. Bleeding: } 5 / 290(1.7 \%) \\
\text { f. Thoracic effusion: } 49 / 290(16.9 \%) \\
\text { g. Esophageal pleural fistula: } \\
1 / 290(0.3 \%) \\
\text { h. Esophageal diverticulum: } \\
\text { 2/290 (0.7\%) }\end{array}$ & $\begin{array}{l}\text { 1. Standard } \\
\text { endoscopy at } \\
3,6 \text { and } 12 \mathrm{~m} \\
\text { and annually } \\
\text { thereafter } \\
\text { 2. CT scan was } \\
\text { performed } \\
\text { the day after } \\
\text { the procedure } \\
\text { to check for } \\
\text { complications }\end{array}$ & DNA & $0 \%$ & $\begin{array}{l}\text { Composite: } \\
89.3 \% \\
(259 / 290)\end{array}$ \\
\hline $\begin{array}{c}78.3 \pm 25.5 \\
(\text { range } 50-130) \\
\min \end{array}$ & $\begin{array}{l}\text { Composite complication rate: DNA } \\
\text { a. Subcutaneous and mediastinal } \\
\text { emphysema: } 8 / 12(66.7 \%) \\
\text { b. Pneumothorax: } 4 / 12(33.3 \%) \\
\text { c. Pneumoperitoneum: } 3 / 12(25 \%) \\
\text { d. Small pleural effusion: } \\
2 / 12(16.7 \%)\end{array}$ & $\begin{array}{l}\text { 1. Standard } \\
\text { endoscopy and } \\
\text { EUS at } 2 \text { and } \\
6 \mathrm{~m} \text {, annually } \\
\text { thereafter } \\
\text { 2. Follow-up } \\
\text { range: } 2-15 \mathrm{~m} \\
\text { (Mean: } 7.1 \mathrm{~m} \text { ) }\end{array}$ & $\begin{array}{l}\text { 1. Esophageal: } \\
100 \%(7 / 7) \\
\text { 2. Gastric: } \\
100 \%(5 / 5)\end{array}$ & $0 \%$ & $\begin{array}{l}\text { 1. Esophageal: } \\
100 \%(7 / 7) \\
\text { 2. Gastric: } \\
100 \%(5 / 5)\end{array}$ \\
\hline 90 & Composite complication rate: $0 \%$ & $\begin{array}{l}\text { Surveillance } \\
\text { endoscopy at } \\
2 \mathrm{~m}\end{array}$ & $\begin{array}{l}\text { Gastric: } \\
100 \%(1 / 1)\end{array}$ & DNA & $\begin{array}{l}\text { Gastric- } 0 \% \\
(0 / 1)\end{array}$ \\
\hline $\begin{array}{c}75.1 \\
(40-100) \mathrm{min}\end{array}$ & $\begin{array}{l}\text { Composite complication rate: } 11.1 \% \\
\text { a. Perforation- } 1 / 18(5.6 \%) \\
\text { b.Pneumoperitoneum - } 1 / 18(5.6 \%)\end{array}$ & $\begin{array}{l}\text { Standard } \\
\text { endoscopy } \\
\text { at } 2 \text { and } 6 \mathrm{~m} \text {, } \\
\text { annually } \\
\text { thereafter }\end{array}$ & $\begin{array}{l}\text { Gastric: } \\
100 \%(18 / 18)\end{array}$ & DNA & DNA \\
\hline
\end{tabular}


Table 1 Continued...

\begin{tabular}{|c|c|c|c|c|c|c|c|}
\hline $\begin{array}{l}\text { Author/Year/ } \\
\text { Location }\end{array}$ & Study type & $\begin{array}{l}\text { Inclusion } \\
\text { criteria }\end{array}$ & $\begin{array}{c}\text { Number } \\
\text { of } \\
\text { subjects }\end{array}$ & $\begin{array}{c}\text { Number } \\
\text { of } \\
\text { lesions }\end{array}$ & $\begin{array}{l}\text { Distribution } \\
\text { of lesions }\end{array}$ & $\begin{array}{c}\text { Mean size of } \\
\text { lesion } \\
\text { (range) }\end{array}$ & $\begin{array}{l}\text { Pathologic } \\
\text { diagnosis }\end{array}$ \\
\hline $\begin{array}{l}\text { Li et al 2014, } \\
\text { China [18] }\end{array}$ & $\begin{array}{l}\text { Retrospective } \\
\text { study }\end{array}$ & $\begin{array}{l}\text { 1. SMT } \\
\text { originating } \\
\text { from MP layer, } \\
\text { confirmed on } \\
\text { EUS } \\
2 . \text { Size: } \\
1.0-5.0 \mathrm{~cm}\end{array}$ & 32 & 32 & Gastric: 32 & $2.3(1.0-5.0)$ & $\begin{array}{l}\text { 1. Leiomyoma } \\
\text { 2. GIST } \\
\text { 3. Glomus tumor } \\
\text { 4. Nerve sheath } \\
\text { tumor } \\
\text { 5. Calcifying } \\
\text { fibrous tumor }\end{array}$ \\
\hline $\begin{array}{l}\text { Zhou et al 2015, } \\
\text { China [19] }\end{array}$ & $\begin{array}{l}\text { Retrospective } \\
\text { study }\end{array}$ & $\begin{array}{l}\text { SMT originating } \\
\text { predominantly } \\
\text { from the MP } \\
\text { layer, confirmed } \\
\text { by CT and EUS }\end{array}$ & 21 & 21 & EGJ: 21 & $2.3(1.0-4.0)$ & $\begin{array}{l}\text { 1. Leiomyoma } \\
\text { 2. GIST }\end{array}$ \\
\hline $\begin{array}{l}\text { Wang et al 2014, } \\
\text { China }[20]\end{array}$ & $\begin{array}{l}\text { Prospective } \\
\text { study }\end{array}$ & $\begin{array}{l}\text { 1. SMT } \\
\text { originating } \\
\text { predominantly } \\
\text { from the MP } \\
\text { layer without } \\
\text { restriction of } \\
\text { extraluminal } \\
\text { growth, } \\
\text { confirmed by } \\
\text { CT and EUS } \\
2 . \text { Size of tumor: } \\
<3.5 \mathrm{~cm}\end{array}$ & 57 & 57 & EGJ: 57 & $2.2(0.6-3.5)$ & $\begin{array}{l}\text { 1. Leiomyoma } \\
\text { 2. GIST } \\
\text { 3. Intramuscular } \\
\text { lipoma } \\
\text { 4. Granular cell } \\
\text { tumor } \\
\text { 5. Schwannoma }\end{array}$ \\
\hline $\begin{array}{l}\text { Tan et al 2015, } \\
\text { China [21] }\end{array}$ & $\begin{array}{l}\text { Retrospective } \\
\text { study }\end{array}$ & $\begin{array}{l}\text { 1. SMT } \\
\text { originating from } \\
\text { the MP layer } \\
\text { confirmed by } \\
\mathrm{CT} \text { and EUS } \\
\text { with confirmed } \\
\text { histologic } \\
\text { diagnosis of } \\
\text { leiomyoma } \\
2 . \text { Size of lesion: } \\
3.5-5.5 \mathrm{~cm} \\
3 . Z \text { ubrod-ECOG } \\
\text { performance } \\
\text { status } 0 \text { or } 1\end{array}$ & 18 & 18 & $\begin{array}{l}\text { Esophageal: } \\
18\end{array}$ & $4.1(3.5-5.3)$ & Leiomyoma \\
\hline $\begin{array}{l}\text { Liu et al 2015, } \\
\text { China [22] }\end{array}$ & Case report & $\begin{array}{l}\text { SMT } \\
\text { originating } \\
\text { predominantly } \\
\text { from the MP } \\
\text { layer, confirmed } \\
\text { by CT and EUS } \\
2 . \text { Size: } \\
5.0 \times 3.0 \mathrm{~cm}\end{array}$ & 1 & 1 & Esophageal: 1 & $5.5 \times 3.5 \times 3.0$ & Leiomyoma \\
\hline $\begin{array}{l}\text { Inoue } \text { et al } \\
2011 \text {, Japan [23] }\end{array}$ & $\begin{array}{l}\text { Prospective } \\
\text { study }\end{array}$ & $\begin{array}{l}\text { Suspected or } \\
\text { confirmed GIST } \\
\text { or leiomyoma, } \\
\text { confirmed on } \\
\text { EUS } \\
2 . \text { Size: }>2.0 \mathrm{~cm} \\
\text { 3. Observed } \\
\text { growth on } \\
\text { follow up }\end{array}$ & 7 & 7 & $\begin{array}{l}\text { Esophageal: } 3 \\
\text { 2. Gastric: } 4\end{array}$ & $\begin{array}{l}\text { 1.Esophagus: } \\
1.5 \times 1.0 \times 0.9 \mathrm{~cm} \\
2 . \text { Gastric: } \\
2.1 \times 1.4 \times 1.1 \mathrm{~cm}\end{array}$ & $\begin{array}{l}\text { Leiomyoma } \\
\text { 2. GIST } \\
\text { 3. Aberrant } \\
\text { pancreas }\end{array}$ \\
\hline
\end{tabular}




\begin{tabular}{|c|c|c|c|c|c|}
\hline $\begin{array}{l}\text { Mean procedure } \\
\text { time (min) } \\
\text { (range) }\end{array}$ & Complications & $\begin{array}{l}\text { Follow-up } \\
\text { interval and } \\
\text { modality }\end{array}$ & $\begin{array}{l}\text { Complete } \\
\text { resection rate } \\
\text { (absolute } \\
\text { number) }\end{array}$ & $\begin{array}{l}\text { Tumor } \\
\text { recurrence } \\
\text { rate }\end{array}$ & $\begin{array}{l}\text { En bloc } \\
\text { resection rate } \\
\text { (absolute } \\
\text { number) }\end{array}$ \\
\hline $\begin{array}{c}51.8 \\
(25-125) \min \end{array}$ & $\begin{array}{l}\text { Composite complication rate: DNA } \\
\text { a. Pneumothorax with subcutaneous } \\
\text { emphysema- } 3 / 32(9.4 \%) \\
\text { b. Pneumoperitoneum - } 6 / 32(18.8 \%) \\
\text { c. Bleeding- } 1 / 32(3.1 \%) \\
\text { d. Pleural effusion- } 3 / 32(9.4 \%) \\
\text { e. Subphrenic infection- } 1 / 32(3.1 \%)\end{array}$ & $\begin{array}{l}\text { 1. Follow-up } \\
\text { range: } 6-32 \mathrm{~m}\end{array}$ & $\begin{array}{l}\text { Gastric: } \\
100 \%(32 / 32)\end{array}$ & $0 \%$ & $\begin{array}{l}\text { Gastric: } \\
100 \%(32 / 32)\end{array}$ \\
\hline $\begin{array}{l}62.9 \\
(45-90) \min \end{array}$ & $\begin{array}{l}\text { Composite complication rate:42.9\% } \\
\text { a. Perforation- } 9 / 21(42.9 \%)\end{array}$ & $\begin{array}{l}\text { 1. Surveillance } \\
\text { endoscopy at } \\
1,3,6 \mathrm{~m} \text { and } \\
\text { EUS at } 3 \mathrm{~m} \text {. } \\
\text { 2. Follow-up } \\
\text { range: } 2-14 \mathrm{~m}\end{array}$ & $\begin{array}{l}\text { EGJ: } \\
100 \%(21 / 21)\end{array}$ & $0 \%$ & $\begin{array}{l}\text { EGJ: } 85.7 \% \\
(18 / 21)\end{array}$ \\
\hline $\begin{array}{c}47 \\
(15-120) \min \end{array}$ & $\begin{array}{l}\text { Composite complication rate: DNA } \\
\text { a. Subcutaneous emphysema- } 12 / 57 \\
\quad(21.0 \%) \\
\text { b. Pneumothorax- } 5 / 57(8.8 \%) \\
\text { c. Pleural effusion- } 2 / 57(3.5 \%) \\
\text { d. Pneumoperitoneum- } 3 / 57(5.2 \%)\end{array}$ & $\begin{array}{l}\text { Follow up } \\
\text { range: } 6-24 \mathrm{~m}\end{array}$ & $\begin{array}{l}\text { EGJ: } \\
100 \%(57 / 57)\end{array}$ & $0 \%$ & $\begin{array}{l}\text { EGJ: } 100 \% \\
(57 / 57)\end{array}$ \\
\hline $75.00 \pm 27.17 \mathrm{~min}$ & $\begin{array}{l}\text { Composite complication rate: } 16.7 \% \\
\text { a. Subcutaneous emphysema: } 1 / 18 \\
\quad(5.6 \%) \text { (conservative management) } \\
\text { b. Chest pain: } 1 / 18(5.6 \%) \\
\text { (conservative management) } \\
\text { c. Mucosal laceration: } 1 / 18(5.6 \%) \\
\text { (required metal stent placement) }\end{array}$ & $\begin{array}{l}\text { 1. Surveillance } \\
\text { endoscopy } \\
\text { or barium } \\
\text { swallow } 1 \text {, } \\
6 \text { and } 12 \mathrm{~m} \\
\text { and annually } \\
\text { thereafter } \\
\text { 2. Mean follow } \\
\text { up: } 10.9 \mathrm{~m}\end{array}$ & $\begin{array}{l}\text { Esophageal: } \\
100 \%(18 / 18)\end{array}$ & $0 \%$ & $\begin{array}{l}\text { Esophageal: } \\
88.9 \%(16 / 18)\end{array}$ \\
\hline $45 \mathrm{~min}$ & Composite complication rate: $0 \%$ & $\begin{array}{l}\text { 1. Surveillance } \\
\text { endoscopy } 3 \text {, } \\
6 \text {, and } 12 \mathrm{~m} \\
\text { 2. Follow up } \\
\text { period: } 12 \mathrm{~m}\end{array}$ & $\begin{array}{l}\text { Esophageal: } \\
100 \%(1 / 1)\end{array}$ & $0 \%$ & $\begin{array}{l}\text { Esophageal: } \\
100 \%(1 / 1)\end{array}$ \\
\hline $\begin{array}{l}\text { 1. Esophagus: } \\
182.7(90-365) \\
\text { min } \\
\text { 2. Gastric- } 129.8 \\
(40-320) \mathrm{min}\end{array}$ & Composite complication rate: $0 \%$ & $\begin{array}{l}\text { 1. Surveillance } \\
\text { endoscopy } \\
\text { on the day } \\
\text { following } \\
\text { procedure } \\
\text { and then } \\
\text { annually }\end{array}$ & $\begin{array}{l}\text { 1. Esophageal: } \\
\text { 100\% }(3 / 3) \\
\text { 2. Gastric: } \\
100 \%(4 / 4)\end{array}$ & DNA & $\begin{array}{l}\text { 1. Esophageal: } \\
100 \%(3 / 3) \\
\text { 2. Gastric: } \\
100 \%(4 / 4)\end{array}$ \\
\hline
\end{tabular}




\section{Hospital stay duration}

\section{Esophageal or EGJ origin}

The mean hospital stay duration varied from 2.7 to 6.0 days. Wang et al described a mean hospital stay duration of 2.7 days with a range of 2-6 days [20]. Zhou et al [19] and Tan et al [21] described mean hospital stay durations of 4.3 and 6.0 days, respectively, with ranges from 3-7 and 4.81-7.19 days.

\section{Gastric origin}

Only one [18] of the three studies [16-18] provided the details of hospital stay length for the planned intervention. Li et al reported a mean hospital stay duration of 3.9 days with a range of 2- 9 days [18].

\section{Mixed}

The mean hospital duration for studies including all tumors, irrespective of their location, varied from 3.2-5.4 days $[9,11,12,14]$.

\section{Follow up}

The follow-up period and modalities varied between different studies as per their protocols. Most of the authors reported using standard endoscopy to confirm the healing at the original site of the resected lesion and also to rule out any residual tumor or local recurrence [8-22]. These surveillance endoscopies were performed every 3-6 months over the first year post procedure and then annually thereafter. Few authors reported using EUS in addition to standard endoscopy as part of the post-procedure surveillance $[9,10,12,15,19]$. In the study by Ye et al, the authors reported using noninvasive tests such as CT scan, abdominal US and chest radiograph on an annual basis to rule out distant metastasis among subjects with GIST, in addition to the use of endoscopy and EUS to rule out local recurrence [9]. The follow-up interval varied widely across different studies and even within a given study, from as short as 1 month [10,12] to as long as 36 months [11]. The details from individual studies are summarized in Table 1.

\section{Outcome}

The authors used two terms to define the outcomes in their respective studies. Complete resection rate (CRR) is used to define the percentage of subjects in whom total resection of the tumor was achieved. En bloc resection rate (EBRR) is used to define the percentage of subjects in whom the tumor was resected with an intact capsule. In our article, we reviewed 703 patients with 736 lesions from 16 individual studies, of which 436 were located in the esophagus, 146 in the EGJ and 154 in the stomach [8-23].
CRR was reported by all studies [8-13,15-23] except one [14]. One study did not reported location-specific CRR for STER of upper GI tract tumors [8]. Thus we could not include the data from this study while calculating locationspecific CRR [8]. The composite CRR for STER of upper GI tract tumors arising from muscularis propria layer was $99.8 \%$ (445/446) [8-13,15-23]. The composite CRR for STER of esophageal, EGJ and gastric SMTs arising from MP layer was $100 \%(208 / 208)[9-13,15,21-23], 100 \%(78 / 78)[19,20]$ and $100 \%(115 / 115)[9-13,15-18,23]$ respectively.

EBRR was reported by all studies [8-16,18-23] except one [17]. Two studies did not reported location specific EBRR for STER of upper GI tract tumors $[8,14]$. Thus we could not combine the data from these studies while calculating location specific EBRR $[8,14]$. The composite EBRR for STER of upper GI tract tumors arising from the muscularis propria layer was $94.6 \%(679 / 718)[8-16,18-23]$. The composite EBRR for STER of esophageal, EGJ and gastric SMTs arising from the muscularis propria layer was $98.6 \%(205 / 208)$ [9-13,15,21-23], $96.2 \%(75 / 78)[19,20]$ and $97.9 \%(95 / 97)[9-13,15,16,18,23]$, respectively. None of the studies reported any tumor recurrence after the initial STER procedure for upper GI tract tumors during the specified follow-up period [8-15,18-22].

\section{Concluding remarks}

STER is a safe, minimally invasive and efficacious alternative approach to surgery for tumors arising from the muscularis propria layer, especially for patients with small tumors $(<3 \mathrm{~cm})$. The composite CRR for STER of upper GI tract tumors arising from the muscularis propria layer was 99.8\% (445/446). The composite CRR for STER of esophageal, EGJ and gastric SMTs arising from the muscularis propria layer was 100\% (208/208), 100\% (78/78) and 100\% (115/115), respectively. The composite EBRR for STER of upper GI tract tumors arising from the muscularis propria layer was $94.6 \%$ (679/718). The composite EBRR for STER of esophageal, EGJ and gastric SMTs arising from the muscularis propria layer was 98.6\% (205/208), 96.2\% (75/78) and 97.9\% (95/97), respectively. The tumor recurrence rate was $0 \%$. The overall reported complication rate for STER was high, but a very small percentage of these required secondary interventions and the majority responded to conservative management. The heterogeneity among the tumor characteristics of patients across the included studies should be taken into consideration when interpreting the above results. Furthermore, as the majority of the studies were from China, the conclusions from our review have potential for bias. Careful selection of candidates by preoperative endoscopy, EUS and crosssectional imaging is important in order to identify benign and exclude potentially malignant tumors. The STER technique is currently restricted to specialized centers and is performed by highly qualified endoscopists. There is a need for head-to-head randomized controlled trials comparing STER to surgery and other available techniques, such as endoscopic full thickness resection, with a long follow up to guide future decisions in 
our approach to upper GI submucosal tumors arising from the muscularis propria.

\section{References}

1. Sakamoto H, Kitano M, Kudo M. Diagnosis of subepithelial tumors in the upper gastrointestinal tract by endoscopic ultrasonography. World J Radiol 2010;2:289-297.

2. Ponsaing LG, Kiss K, Hansen MB. Classification of submucosal tumors in the gastrointestinal tract. World $J$ Gastroenterol 2007;13:3311-3315.

3. Lee IL, Lin PY, Tung SY, Shen CH, Wei KL, Wu CS. Endoscopic submucosal dissection for the treatment of intraluminal gastric subepithelial tumors originating from the muscularis propria layer. Endoscopy 2006;38:1024-1028.

4. American Gastroenterological Association Institute. American Gastroenterological Association Institute medical position statement on the management of gastric subepithelial masses. Gastroenterology 2006;130:2215-2216.

5. Shi Q, Zhong YS, Yao LQ, Zhou PH, Xu MD, Wang P. Endoscopic submucosal dissection for treatment of esophageal submucosal tumors originating from the muscularis propria layer. Gastrointest Endosc 2011;74:1194-1200.

6. De Vogelaere K, Hoorens A, Haentjens P, Delvaux G. Laparoscopic versus open resection of gastrointestinal stromal tumors of the stomach. Surg Endosc 2013;27:1546-1554.

7. Białek A, Wiechowska-Kozłowska A, Pertkiewicz J, et al. Endoscopic submucosal dissection for treatment of gastric subepithelial tumors (with video). Gastrointest Endosc 2012;75:276-286.

8. Lu J, Jiao T, Zheng M, Lu X. Endoscopic resection of submucosal tumors in muscularis propria: the choice between direct excavation and tunneling resection. Surg Endosc 2014;28:3401-3407.

9. Ye LP, Zhang Y, Mao XL, Zhu LH, Zhou X, Chen JY. Submucosal tunneling endoscopic resection for small upper gastrointestinal subepithelial tumors originating from the muscularis propria layer. Surg Endosc 2014;28:524-530.

10. Xu MD, Cai MY, Zhou PH, et al. Submucosal tunneling endoscopic resection: a new technique for treating upper GI submucosal tumors originating from the muscularis propria layer (with videos). Gastrointest Endosc 2012;75:195-199.

11. Zhang C, Hu JW, Chen T, et al. Submucosal tunneling endoscopic resection for upper gastrointestinal multiple submucosal tumors originating from the muscular propria layer: a feasibility study. Indian J Cancer 2015;51(Suppl 2):e52-e55.

12. Wang H, Tan Y, Zhou Y, et al. Submucosal tunneling endoscopic resection for upper gastrointestinal submucosal tumors originating from the muscularis propria layer. Eur J Gastroenterol Hepatol 2015;27:776-780.
13. Chen H, Xu Z, Huo J, Liu D. Submucosal tunneling endoscopic resection for simultaneous esophageal and cardia submucosal tumors originating from the muscularis propria layer (with video). Dig Endosc 2015;27:155-158.

14. Chen T, Zhang C, Yao LQ, et al. Management of the complications of submucosal tunneling endoscopic resection for upper gastrointestinal submucosal tumors. Endoscopy 2016;48:149-155.

15. Liu BR, Song JT, Kong LJ, Pei FH, Wang XH, Du YJ. Tunneling endoscopic muscularis dissection for subepithelial tumors originating from the muscularis propria of the esophagus and gastric cardia. Surg Endosc 2013;27:4354-4359.

16. Jeong ES, Hong SJ, Han JP, Kwak JJ. Submucosal tunneling endoscopic resection of a leiomyoma originating from the muscularis propria of the gastric cardia (with video). Korean $J$ Gastroenterol 2015;66:340-344.

17. Lu J, Zheng M, Jiao T, Wang Y, Lu X. Transcardiac tunneling technique for endoscopic submucosal dissection of gastric fundus tumors arising from the muscularis propria. Endoscopy 2014;46:888-892.

18. Li QL, Chen WF, Zhang C, et al. Clinical impact of submucosal tunneling endoscopic resection for the treatment of gastric submucosal tumors originating from the muscularis propria layer (with video). Surg Endosc 2015;29:3640-3646.

19. Zhou DJ, Dai ZB, Wells MM, Yu DL, Zhang J, Zhang L. Submucosal tunneling and endoscopic resection of submucosal tumors at the esophagogastric junction. World J Gastroenterol 2015;21:578-583.

20. Wang XY, Xu MD, Yao LQ, et al. Submucosal tunneling endoscopic resection for submucosal tumors of the esophagogastric junction originating from the muscularis propria layer: a feasibility study (with videos). Surg Endosc 2014;28:1971-1977.

21. Tan Y, Lv L, Duan T, et al. Comparison between submucosal tunneling endoscopic resection and video-assisted thoracoscopic surgery for large esophageal leiomyoma originating from the muscularis propria layer. Surg Endosc 2016;30:3121-3127.

22. Liu H, Wei LL, Zhang YZ, et al. Submucosal tunnelling endoscopic resection (STER) for the treatment of a case of huge esophageal tumor arising in the muscularis propria: a case report and review of literature. Int J Clin Exp Med 2015;8:15846-15851.

23. Inoue H, Ikeda H, Hosoya T, et al. Submucosal endoscopic tumor resection for subepithelial tumors in the esophagus and cardia. Endoscopy 2012;44:225-230.

24. Chen T, Zhou PH, Chu Y, et al. Long-term outcomes of submucosal tunneling endoscopic resection for upper gastrointestinal submucosal tumors. Ann Surg 2016 Jan 19. [Epub ahead of print].

25. Yoshizumi F, Yasuda K, Kawaguchi K, Suzuki K, Shiraishi N, Kitano S. Submucosal tunneling using endoscopic submucosal dissection for peritoneal access and closure in natural orifice transluminal endoscopic surgery: a porcine survival study. Endoscopy 2009;41:707-711.

26. Inoue H, Minami H, Kobayashi Y, et al. Peroral endoscopic myotomy (POEM) for esophageal achalasia. Endoscopy 2010;42:265-271. 